\title{
VARIABILIDAD ESPACIAL DELSUELO Y SU RELACION CON EL RENDIMIENTO DE MANGO (Mangifera indica L.) ${ }^{1}$
}

\author{
CESAR AUGUSTO VALBUENACALDERÓN² ${ }^{2}$ LUÍS JOEL MARTÍNEZ MARTÍNEZ ${ }^{3}$ RAMÓN GIRALDOHENAO
}

RESUMEN- Se realizó un muestreo en una red regular de 31 puntos ubicados con GPS y con base en análisis geoestadísticos, se estudió la variabilidad espacial de algunas propiedades del suelo y de la topografía con el fin de establecer su incidencia en el rendimiento de un cultivo de mango. Las propiedades edáficas y el rendimiento presentaron un patrón de distribución espacial que varía de manera considerable dentro del lote. El rango de los modelos de semivarianza ajustados varió entre 10 y 192 metros. Con excepción del pH y del Ca las relaciones efecto pepita/meseta fueron menores del 30\%, es decir, que en general hay precisión en las predicciones debido a que los procesos espaciales considerados son explicados en su mayoría por la variación estructural. K, Ca, $\mathrm{CIC}$, pendiente del terreno, relación $\mathrm{Ca}+\mathrm{Mg} / \mathrm{K}$ y altitud fueron las variables que mayor influencia tuvieron en el rendimiento. El método aplicado es útil para definir zonas de manejo dentro de los lotes y algunos criterios como la altitud y la pendiente son aplicables para zonificación de áreas mayores calculándolos a partir de un modelo digital de elevación.

Términos para indexación: variabilidad espacial, geoestadística, cultivo de mango.

\section{SPATIAL VARIABILITY OF SOIL PROPERTIES AND YIELD RELATIONSHIP IN A MANGO CROP} (Mangifera indica L.)

\begin{abstract}
In a regular grid with 31 points located with GPS, samples were taken and based on geostatistics analysis the spatial variability of soil properties and their relationships with yield of a mango crop were studied. It was found that soil properties and crop yield presented a high, within-field, spatial variability. The range of the semi variance models varied from 10 to 192 meters, with the exception of $\mathrm{pH}$ and $\mathrm{Ca}$, the adjusted models showed nugget/sill fraction lower than $30 \%$, thus indicating that most of the process is explained by the structural variation and then better interpolation results could be expected. $\mathrm{K}, \mathrm{Ca}, \mathrm{CIC}, \mathrm{Ca}+\mathrm{Mg} / \mathrm{K}$ fraction, slope, and altitude were the most important factors affecting crop yield. The studied method in this research is useful to define management zones within fields and some used criteria like altitude and slope are important for the zoning of larger areas and can be computed from a digital elevation model.
\end{abstract}

Index Terms: spatial variability, geostatistics, mango crop.

\section{INTRODUCCIÓN}

La producción de los cultivos fluctúa dentro de los lotes, como consecuencia de la variación que presentan diversos factores, entre ellos las propiedades del suelo (Godwin \& Miller, 2003; Taylor et al., 2003) y la topografía (Kravchenko et al., 2005; Reyniers et al., 2006). Conocer la variabilidad de los factores mencionados y su relación con la producción, es la base de la agricultura de precisión y es fundamental para establecer sistemas de producción más sostenibles y eficientes en el uso de los insumos, por ejemplo, mediante aplicaciones diferenciales de fertilizantes.

En Colombia el mango es un frutal de gran importancia, ocupa una extensión total de 8300 hectáreas, con una producción media de 8-10 toneladas/hectárea, tiene gran aceptación en el mercado nacional e internacional por lo cual se considera como un cultivo promisorio (Miranda, 2001). Las exportaciones colombianas de mango han mostrado un continuo ascenso desde 1997, alcanzando 14.000 toneladas en el año 2001 (Yahia et al., 2006). Mejorar el manejo actual de la aplicación generalizada de insumos, considerando la variabilidad interna de los lotes, es una alternativa que puede repercutir positivamente en la competitividad del cultivo.

Para el estudio de la variabilidad espacial de las propiedades del suelo se ha acudido con frecuencia al uso de análisis geoestadísticos (Goovaerts, 1999). Estas técnicas se han empleado para el mejoramiento de los levantamientos de suelos (Webster, 1985), el estudio de la génesis de los suelos y de pureza de unidades de mapeo (Wilding \& Drees, 1983) y en la determinación de la degradación de los suelos (Martínez \& Zinck, 1994; Martínez \& Zinck, 2004). La geoestadística trata con variables medidas en una región con continuidad espacial, se fundamenta en el estudio de la autocorrelación espacial, es decir, asume que las observaciones de dos sitios serán más similares en la medida en que estos sean más cercanos (Cressie, 1993). El objetivo del presente trabajo fue caracterizar la distribución espacial de variables edáficas dentro de un cultivo de mango (Mangifera indica L.) y establecer su relación con el rendimiento del cultivo. Para lograr estos objetivos se construyeron, con base en métodos geoestadísticos, mapas de distribución espacial de cada una de las características evaluadas y de componentes

\footnotetext{
1(Trabajo 034-08). Recibido el: 19-09-2008. Aceptado para publicación el: 30-01-2008.

2Ingeniero Agrónomo, Universidad Nacional de Colombia, Bogota. e-mail: cavalbuenac@ unal.edu.co.

${ }^{3}$ Profesor asociado, Facultad de Agronomía, Universidad Nacional de Colombia, Bogota. e-mail: 1jmartinezm@unal.edu.co.

${ }^{4}$ Profesor asociado, Facultad de Ciencias, Universidad Nacional de Colombia, Bogota. e-mail:rgiraldoh@unal.edu.co.
} 
principales (Peña, 2002) generados a partir de las variables de mayor correlación con el rendimiento.

\section{MATERIALES Y MÉTODOS}

La investigación se realizó en el municipio de La Mesa, departamento de Cundinamarca, Colombia (435'41" N, 74²7'50" W), en un cultivo de mango (Mangifera indica L.), variedad Fairchild, de 8 años de edad, con una extensión de 5 hectáreas, ubicado en un Typic Dystrudept. El clima de la zona se clasifica como Am según el sistema Köeppen, es húmedo con un período seco, con temperatura que varía entre $23^{\circ} \mathrm{C}$ y $26^{\circ} \mathrm{C}$ y precipitación promedio anual de $2500 \mathrm{~mm}$. Estas condiciones se consideran apropiadas para el cultivo del mango (Galán, 1999).

El muestreo se efectuó en una red de 31 puntos, ubicando cada uno de ellos por medio de un equipo GPS. En cada sitio se tomaron 5 submuestras de suelo, una en el punto y cuatro separadas a $1 \mathrm{~m}$ de éste, luego se mezclaron homogéneamente y se conformaron muestras compuestas que fueron analizadas en laboratorio según los métodos del Instituto Geográfico Agustín Codazzi (IGAC, 1990). Se midió el carbono orgánico utilizando el método Walkley-Black con valoración colorimétrica. El nitrógeno fue calculado a través del valor del carbono orgánico. La capacidad de intercambio catiónico (CIC), se determinó al desplazar el $\mathrm{NH}_{4}^{+}$intercambiado con $\mathrm{NaCl} 1 \mathrm{M}$ y se valoró volumétrica. $\mathrm{El} \mathrm{pH}$ se evaluó con el potenciómetro en relación suelo: agua 1:1. Los cationes $\mathrm{K}, \mathrm{Na}, \mathrm{Mg}$, y Ca se extrajeron con acetato de amonio $1 \mathrm{~N}, \mathrm{pH} 7$ y se practicó la valoración por espectrometría de absorción atómica. El P se determinó mediante el método Bray II y el aluminio se estimó con $\mathrm{KCl} 1 \mathrm{M}$. En cada punto se tomó la altura sobre el nivel del mar, se halló el rendimiento contando el número total de frutos por árbol y se evaluó la altura del árbol.

Los datos obtenidos se transfirieron al software ArcGis 9.2 (Booth, 2000) y con la ayuda de un Modelo Digital de Elevación se calculó la pendiente del terreno y se simuló el relieve. Con el software R (R Development Core Team, 2005) se calcularon las estadísticas descriptivas y se hicieron gráficos exploratorios con el propósito de determinar el comportamiento global de las variables dentro del lote, de identificar la presencia de estacionariedad en la media y de comprobar empíricamente la normalidad de los datos. Se obtuvieron modelos de semivarianza experimentales por el método de los momentos (Cressie, 1993) y se les ajustaron modelos teóricos por máxima verosimilitud (Diggle \& Ribeiro, 2000). Mediante el método kriging ordinario (Cressie, 1993) se efectuaron las predicciones espaciales en sitios no observados. Con el objetivo de describir de manera general la influencia de las variables edáficas sobre el rendimiento, se realizó un análisis de componentes principales (Peña, 2002) basado en las variables de mayor correlación. Con los factores resultantes se construyeron mapas de distribución espacial siguiendo el mismo procedimiento geoestadístico empleado con las variables originales.

\section{RESULTADOS Y DISCUSIÓN}

Comparando los promedios de las propiedades del suelo consideradas (Tabla 1), con los requerimientos del mango (Sukthumrong et al., 2000) puede decirse que en general el suelo presenta contenidos medios de $\mathrm{P}, \mathrm{Ca}, \mathrm{Mg}, \mathrm{Fe}, \mathrm{Cu}, \mathrm{N}, \mathrm{Zn}$ para ese cultivo y que sólo los valores de $\mathrm{K}$ y Mn resultan bajos. Los promedios del pH y la CIC están dentro los límites considerados como adecuados para el cultivo (Galán, 1999). El aluminio muestra valores que no son tóxicos para la planta. De otro lado con relación a las variables agronómicas hay dos aspectos relevantes a mencionar: La altura promedio (Tabla 1) es coherente con la edad del cultivo y el rango del rendimiento (Tabla 1) es muy amplio, indicando que hay condiciones diferenciales dentro del lote.

Los coeficientes de variación obtenidos (Tabla 1) son similares a los obtenidos por Wilding \& Drees (1983). Es importante mencionar que la variabilidad espacial de las propiedades del suelo depende de la escala y generalmente es mayor si se pasa de pedones a polipedones o unidades de mapeo (Wilding \& Drees, 1983). Aunque los coeficientes de variación (Tabla 1) son relativamente bajos, excepto para el $\mathrm{Al}$ y Mn, y por tanto indican homogeneidad, análisis posteriores muestran que el patrón de distribución espacial varía en forma considerable.

Los coeficientes de correlación (Tabla 2) indican que el rendimiento está directamente relacionado con altitud, altura del árbol, CIC y con el contenido de $\mathrm{K}$ e inversamente asociado con la pendiente del terreno y con la relación $\mathrm{Ca}+\mathrm{Mg} / \mathrm{K}$. El efecto negativo de la pendiente puede indicar la incidencia de la profundidad del suelo y del contenido de humedad que son menores en las partes de mayor pendiente debido a la incidencia de procesos erosivos y presentan valores superiores las partes más planas y cóncavas por acumulación de materiales. Por su parte la incidencia de la altitud puede asociarse a cambios en las condiciones microclimáticas. Kravchenko \& Bullock; (2000) concluyen que la altitud, la pendiente y algunas características edáficas inciden en los rendimientos, pero el efecto varía de un lote a otro dependiendo de las condiciones climáticas y de otros factores aún no estudiados. También se encontró un efecto positivo de la CIC y del $\mathrm{K}$ en el rendimiento posiblemente asociado a mayor contenido de materia orgánica y su efecto en las condiciones de fertilidad y físicas del suelo. Jiang \& Thelen (2004) encontraron que la pendiente, la altura, la posición geomorfológica, la textura, el pH y la saturación de bases explicaron hasta el $85 \%$ de la variación del rendimiento del sistema de cultivo de maíz-soya. Vrindts et al. (2003) encontraron relación entre la topografía y la textura en los rendimientos de los cereales.

Otras correlaciones indicaron una asociación positiva del $\mathrm{pH}$ con la relación $\mathrm{Ca}+\mathrm{Mg} / \mathrm{K}$, boro, carbono orgánico, fósforo y calcio e inversa con hierro y con cobre (Tabla 2). $\mathrm{Ca}+\mathrm{Mg} / \mathrm{K}$ se relacionó en forma positiva con calcio y en forma negativa con la altura del árbol y con los contenidos de cobre, potasio y hierro. Por su parte el calcio se relacionó en forma positiva con el fósforo y boro y en forma negativa con hierro y cobre. Los resultados anteriores parecen indicar una deficiencia de potasio ocasionada 
por los contenidos bajos de ese nutrimento en gran parte del lote y por los elevados contenidos de Ca que pueden afectar la absorción de $\mathrm{K}$ aún cuando se encuentre en cantidades apropiadas en el suelo. En investigaciones efectuadas en cultivos de naranja en Brasil (Fidalski et al., 2000) encontraron correlaciones negativas de la producción con la relación $\mathrm{Ca}+\mathrm{Mg} / \mathrm{K}$ y lo atribuyeron a antagonismo entre iones, por su parte McLean et al. (1983) consideran que no existen unos valores apropiados de esa relación para los cultivos, y que el efecto se debe a deficiencias o excesos de los nutrimentos. de modelos

Estimación de parámetros geoestadísticos y ajuste

De acuerdo con los rangos de los modelos de semivarianza ajustados (Tabla 3) existe fuerte dependencia espacial en las variables $\mathrm{pH}, \mathrm{Ca}$ y $\mathrm{Cu}$ (rangos mayores a $100 \mathrm{~m}$ ), moderada correlación espacial para CIC, Fe, altitud y rendimiento y baja asociación espacial para CO, K, P y B (rangos menores a $30 \mathrm{~m}$ ). Esta variación espacial depende de los procesos y factores formadores del suelo y del manejo a que se haya sometido, por lo tanto pueden ser rangos menores como los reportados (Silva et al., 2008), y por Muñoz et al. (2006) o rangos mayores como los encontrados por Funakawa et al. (2006). Con excepción del pH y del Ca, la relación efecto pepita/meseta fue menor al $30 \%$ indicando con esto que la distancia de muestreo considerada con la red es apropiada para la mayoría de las variables y que las predicciones obtenidas por el método kriging son confiables.

\section{Predicción espacial y mapas de distribución}

En la figura 1 se presenta el mapa de distribución espacial del rendimiento y de las variables que mayor correlación presentaron con el mismo. El mapa de rendimiento indica que la parte baja del lote presenta menor producción y que hay un aumento hacia las partes altas del mismo, espacialmente en dirección sur-norte.

De acuerdo con el mapa de altitud (Figura 1) el lote presenta un relieve quebrado que fluctúa entre $720 \mathrm{~m}$ y $760 \mathrm{~m}$. El Ca presenta los mayores valores en la parte baja del lote y su contenido en el suelo disminuye hacia las partes de mayor altura (Figura 1), por su parte el K presenta los contenidos más bajos hacia la parte baja y los mayores hacia la parte central del lote. Con base en lo anterior puede decirse que en la parte sur del lote, se obtuvo el menor rendimiento y que éste está asociado a contenidos altos de $\mathrm{Ca}$ y bajos de $\mathrm{K}$. La zona sur-oriental tiene altitud media, menores contenidos de Ca que la anterior y mayores de K, allí la producción fue superior. La zona norte tiene la mayor altitud, presenta contenidos bajos de Ca y bajos o medios de $\mathrm{K}$, allí la producción fue mayor.

Si se analiza la variación espacial de estas propiedades se encuentra que existen áreas dentro del lote que tienen deficiencias nutricionales y otras que por el contrario presentan condiciones apropiadas e incluso en algunos casos excesivas. Por ejemplo el $\mathrm{pH}$ cuyo valor medio (Tabla 1) es apropiado para el cultivo muestra en algunas zonas valores o muy bajos (menores a 5.5) o muy altos (mayores a 7.2) que resultan limitantes (Galán, 1999). En el caso del P, Fe, Cu, $\mathrm{N}$ aunque los promedios se califican como moderados, en algunas partes del lote sus magnitudes son bajas o muy bajas y consecuentemente deficientes para el cultivo. En algunas áreas los contenidos de $\mathrm{Ca}, \mathrm{Fe}, \mathrm{Mg}$, pueden ser considerados como muy altos para el cultivo a pesar de que como se mencionó anteriormente sus valores medios se consideran apropiados.

Estos resultados son muy interesantes ya que permiten definir zonas de manejo dentro de los lotes con el fin de aplicar en forma más eficiente los nutrimentos de manera en que se pueda llegar al manejo específico por sitio o a la agricultura de precisión.

\section{Análisis de componentes principales}

De acuerdo con los valores propios (Tabla 4) los dos primeros componentes principales explican el $63.78 \%$ de la variabilidad total contenida en las diez variables consideradas para este análisis. Estos dos serán considerados para llevar a cabo el análisis geoestadístico factorial.

Los vectores propios (Tabla 5) indican que el componente principal uno (CP1) explica con mayor fuerza lo que ocurre de manera directa con las variables $\mathrm{Fe}, \mathrm{Cu}$ y altura del árbol y de forma inversa lo que sucede con $\mathrm{pH}, \mathrm{B}, \mathrm{Ca}, \mathrm{P}, \mathrm{CO}$ y K ; en el caso del componente principal dos (CP2) se revela en gran medida lo que sucede con $\mathrm{CIC}, \mathrm{K}$ y $\mathrm{CO}$ en sentido directo y $\mathrm{Ca}$ en el inverso. De acuerdo con esto, los sitios del lote con valores positivos en el componente principal uno estarán asociados a valores por encima del promedio en $\mathrm{Fe}, \mathrm{Cu}$ y altura del árbol y a magnitudes por debajo de la media en $\mathrm{pH}, \mathrm{B}, \mathrm{Ca}, \mathrm{P}, \mathrm{CO}$ y K, los que tengan valores positivos en el componente dos, corresponderán a aquellos con CIC, K y CO por encima del promedio global y valores por debajo de la media en $\mathrm{Ca}$.

La observación simultánea de los mapas de distribución espacial del rendimiento (Figura 1) y del CP1 (Figura 2), permite deducir que el primero se ve favorecido por magnitudes por encima de $111.71 \mathrm{mg} \mathrm{Kg}^{-1}$ de $\mathrm{Fe}, 1.46 \mathrm{mg} \mathrm{Kg}^{-1}$ de $\mathrm{Cu}$, y de 383.87 $\mathrm{cm}$ en la altura del árbol y además que valores por debajo de 5.59 de $\mathrm{pH}, 0.33 \mathrm{mg} \mathrm{Kg}^{-1}$ de B, $16.21 \mathrm{cmol} . \mathrm{Kg}^{-1}$ de Ca, $19.3 \mathrm{mg} \mathrm{Kg}^{-1} \mathrm{de}$ $\mathrm{Py} \mathrm{de} 2.23 \%$ para el CO pueden ser favorables para el rendimiento del cultivo estudiado. Esta conclusión resulta del hecho de que en la zona norte y en el borde nororiental del área de estudio donde se da el mayor rendimiento (Figura 1) se presentan los valores más altos del CP1 (Figura 2).

La distribución espacial del CP2 (Figura 2) resulta heterogénea dentro del lote, se pueden observar varias "manchas" o "parches" de valores altos combinándose con valores bajos y tiene un comportamiento muy similar al presentado de manera individual por el CIC, altura árbol y carbono orgánico (Figuras no incluidas). Aunque estas variables sean importantes para el desarrollo del cultivo, el resultado encontrado con el mapa del CP2 demuestra que no son de las más relevantes en la explicación del comportamiento espacial del rendimiento. 
TABLA 1- Estadísticas descriptivas de las variables estudiadas. La Mesa, Cundinamarca, 2008.

\begin{tabular}{|c|c|c|c|c|c|c|}
\hline VARIABLE & Unidades & Mín. & Máx. & SD & $\mathrm{CV}(\%)$ & $\overline{\text { Promedio }}$ \\
\hline Reacción del suelo $(\mathrm{pH})$ & & 4,58 & 7,61 & 0,94 & 16,82 & 5,59 \\
\hline Carbono orgánico (C0) & $\begin{array}{c}1--14 \\
\%\end{array}$ & 0,95 & 3,61 & 0,7 & 31,39 & 2,23 \\
\hline Nitrógeno (N) & $\%$ & 0,08 & 0,31 & 0,061 & 32,11 & 0,19 \\
\hline Fósforo (P) & $\mathrm{mg} \cdot \mathrm{Kg}^{-1}$ & 3,5 & 55,7 & 14,29 & 74,04 & 19,3 \\
\hline Potasio (K) & $\mathrm{cmol} . \mathrm{Kg}^{-1}$ & 0,21 & 1,09 & 0,22 & 59,46 & 0,37 \\
\hline Calcio (Ca) & $\mathrm{cmol} . \mathrm{Kg}^{-1}$ & 7,18 & 44,26 & 7,34 & 45,28 & 16,21 \\
\hline $\begin{array}{l}\text { Suma de bases } \\
\text { de cambio (Bases) }\end{array}$ & cmol. $\mathrm{Kg}^{-1}$ & 14,65 & 46 & 6,73 & 31,73 & 21,21 \\
\hline Magnesio (Mg) & cmol. $\mathrm{Kg}^{-1}$ & 1,08 & 8,48 & 1,68 & 42,97 & 3,91 \\
\hline $\mathrm{Ca}+\mathrm{Mg} / \mathrm{K}$ & & 21,02 & 217,86 & 35,31 & 61,92 & 65,32 \\
\hline Capacidad de Cambio & cmol. $\mathrm{Kg}^{-1}$ & 14,8 & 26,8 & 2,52 & 12,06 & 20,89 \\
\hline \multicolumn{7}{|l|}{ Cationico (CIC) } \\
\hline Boro (B) & $\mathrm{mg} \cdot \mathrm{Kg}^{-1}$ & 0,14 & 1,08 & 0,18 & 54,55 & 0,33 \\
\hline Cobre $(\mathrm{Cu})$ & $\mathrm{mg} \cdot \mathrm{Kg}^{-1}$ & 0,72 & 2,53 & 0,46 & 31,51 & 1,46 \\
\hline Hierro (Fe) & $\mathrm{mg} \cdot \mathrm{Kg}^{-1}$ & 14 & 241 & 59,39 & 53,16 & 111,71 \\
\hline Manganeso (Mn) & $\mathrm{mg} \cdot \mathrm{Kg}^{-1}$ & 2,42 & 41,89 & 7,05 & 88,35 & 7,98 \\
\hline Altitud (Alt) & msnm & 674 & 728 & 13,02 & 56,35 & 702,74 \\
\hline Pendiente (Pn) & $\%$ & 8 & 89 & 17,29 & 40,25 & 28,12 \\
\hline Producción (Pr) & Frutos/árbol & 0 & 420 & 112 & 75,16 & 149 \\
\hline Altura árbol & $\mathrm{cm}$ & 240 & 590 & 85,39 & 22,24 & 383,87 \\
\hline
\end{tabular}

TABLA 2- Coeficientes de correlación significativos entre algunas variables.

\begin{tabular}{llllllllll}
\hline Variable & $\mathbf{C I C}$ & $\mathbf{P}$ & $\mathbf{C u}$ & $\mathbf{C O}$ & $\mathbf{B}$ & $\mathbf{F e}$ & $\mathbf{C a}+\mathbf{M g} / \mathbf{K}$ & $\mathbf{C a}$ & $\mathbf{P r}$ \\
\hline $\mathbf{p H}$ & 0,42 & $-0,58^{*}$ & $0,47^{*}$ & $0,77^{*}$ & $-0,68^{*}$ & 0,44 & $0,77^{*}$ & & \\
$\mathbf{K}$ & $0,55^{*}$ & & $0,54^{*}$ & $0,51^{*}$ & & $-0,49^{*}$ & & 0,35 & \\
$\mathbf{C I C}$ & & & & $0,74^{*}$ & 0,42 & & & & 0,31 \\
$\mathbf{P}$ & & & $-0,44$ & & 0,42 & $-0,50^{*}$ & & 0,37 & \\
$\mathbf{C u}$ & & & & & $-0,43$ & $0,79^{*}$ & $-0,36$ & $-0,60^{*}$ & \\
$\mathbf{B}$ & & & & $0,70^{*}$ & & $-0,45^{*}$ & & $0,57^{*}$ & \\
$\mathbf{F e}$ & & & & & & & $-0,47^{*}$ & $-0,67^{*}$ & \\
$\mathbf{C a}+\mathbf{M g} / \mathbf{K}$ & & & & & & & $0,75^{*}$ & $-0,37$ \\
Alt & & & & & & $-0,41$ & & $0,49^{*}$ \\
Pn & & & & & & & & $-0,43$ \\
Altura árbol & & & & & & & & $0,51^{*}$ \\
\hline
\end{tabular}

*Significante al nivel 0.01, las demás son significantes al nivel 0.05 .

TABLA 3 - Modelos de semivarianza ajustados y parámetros estimados. La Mesa, Cundinamarca, 2008.

\begin{tabular}{|c|c|c|c|c|}
\hline \multirow[t]{2}{*}{ VARIABLE } & \multirow[t]{2}{*}{ Modelo } & \multicolumn{3}{|c|}{ Parámetros estimados } \\
\hline & & Nugget & Sill & Rango (m) \\
\hline pH & Exponencial & 0,745 & 0,584 & 192,4 \\
\hline $\mathrm{CO}$ & Gaussiano & 0 & 0,504 & 18,1 \\
\hline $\mathrm{Ca}$ & Exponencial & 0,120 & 0,166 & 139,0 \\
\hline $\mathbf{K}$ & Circular & 0,007 & 0,057 & 10,0 \\
\hline CIC & Exponencial & 0,670 & 8,750 & 37,7 \\
\hline $\mathbf{P}$ & Efecto pepita & 12,240 & 194,40 & 2,2 \\
\hline $\mathrm{Cu}$ & Exponencial & 0,039 & 0,318 & 130,0 \\
\hline $\mathrm{Fe}$ & Circular & 1051 & 4178 & 41,7 \\
\hline B & Gaussiano & 0 & 0,040 & 10,0 \\
\hline Pr. & Exponencial & 0 & 10000 & 50,0 \\
\hline $\begin{array}{l}\text { Alt. } \\
\text { Componente } 1\end{array}$ & $\begin{array}{l}\text { Circular } \\
\text { Gaussiano }\end{array}$ & $\begin{array}{l}1051 \\
0\end{array}$ & $\begin{array}{l}6178 \\
7,120\end{array}$ & $\begin{array}{l}41,7 \\
70,0\end{array}$ \\
\hline Componente 2 & Gaussiano & 0 & 3,030 & 21,6 \\
\hline
\end{tabular}

TABLA 4 - Valores propios y porcentajes de variabilidad explicada por los componentes principales generados a partir de la matriz de correlación de 10 de las variables consideradas. La Mesa, Cundinamarca, 2008.

\begin{tabular}{cccc}
\hline Componentes & Valor propio & $\begin{array}{c}\text { Porcentaje de } \\
\text { variación }\end{array}$ & Porcentaje acumulado \\
\hline 1 & 2.07 & 39.30 & 39.30 \\
2 & 1.64 & 24.48 & 63.78 \\
3 & 1.16 & 12.23 & 76.02 \\
4 & 0.83 & 6.33 & 82.35 \\
5 & 0.73 & 4.93 & 87.28 \\
6 & 0.64 & 3.76 & 91.05 \\
7 & 0.56 & 2.89 & 93.94 \\
8 & 0.54 & 2.72 & 96.66 \\
9 & 0.43 & 1.71 & 98.38 \\
10 & 0.33 & 1.62 & 100.00 \\
\hline
\end{tabular}


TABLA 5- Ponderaciones de las variables para las dos componentes en los que se presentó la mayor variación. La Mesa, Cundinamarca, 2008

\begin{tabular}{ccc}
\hline Variable & Componente 1 & Componente 2 \\
\hline Alt. & 0.14 & 0.31 \\
pH & -0.44 & 0.07 \\
CO & -0.26 & 0.40 \\
Ca & -0.38 & -0.17 \\
K & -0.18 & 0.44 \\
CIC & 0 & 0.50 \\
$\mathbf{P}$ & -0.29 & 0.09 \\
Cu & 0.35 & 0.23 \\
Fe & 0.37 & 0.24 \\
B & -0.40 & 0.20 \\
\hline
\end{tabular}
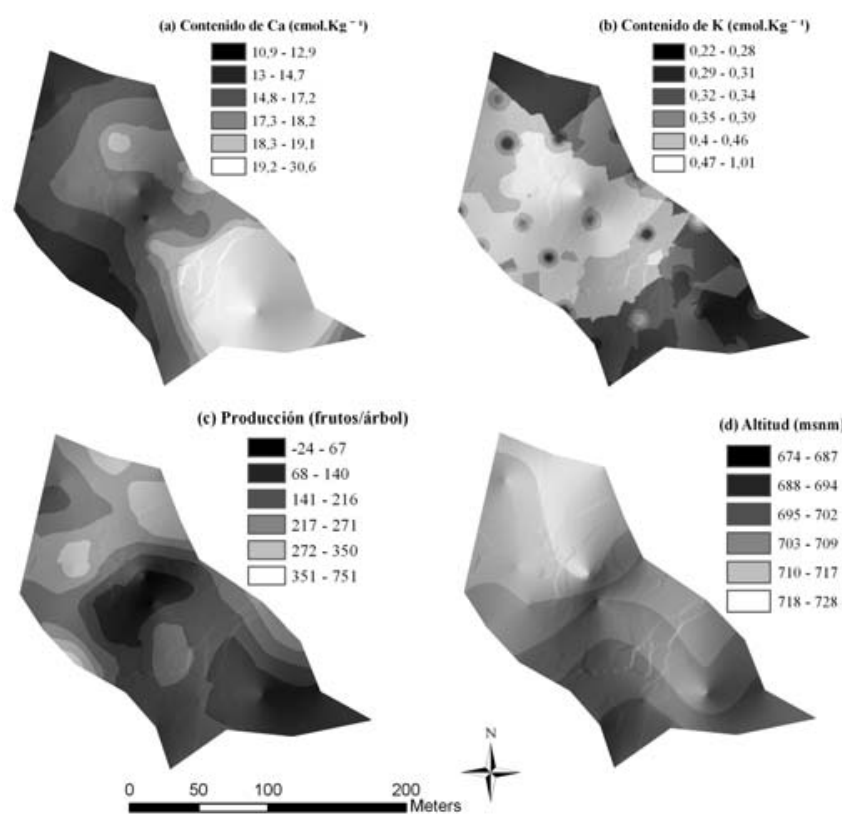

FIGURA 1 - Distribuición espacial de las variables: contenido de $\mathrm{Ca}$ (a), contenido de $\mathrm{K}(\mathrm{b})$, producción de frutos (c), altitud (d). La Mesa, Cundinamarca.

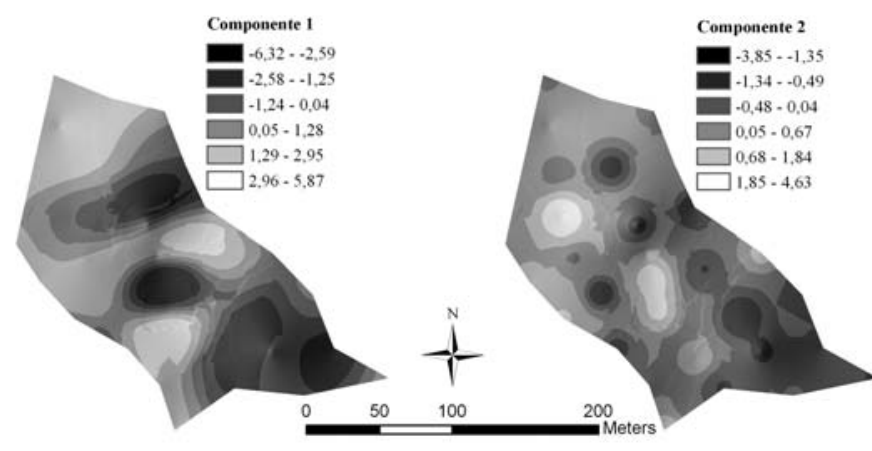

FIGURA 2 - Distribuición espacial del los componentes principales. La Mesa, Cundinamarca,2008.

\section{CONCLUSIONES}

Las propiedades edáficas evaluadas y la producción del cultivo del mango se distribuyen dentro del lote estudiado con una estructura espacial bien definida. La variabilidad espacial de las propiedades químicas del suelo y de la topografía dentro del lote de mango es alta, al igual que el rendimiento del cultivo. Los análisis espaciales permitieron conocer la distribución y la variabilidad de las propiedades del suelo y su relación con la producción. Aunque los valores medios de algunas propiedades del suelo, para todo el lote, se pueden considerar apropiados para el cultivo del mango, el análisis espacial demostró que dentro del lote hay áreas con deficiencia de nutrimentos y otras incluso, con exceso de los mismos lo cual es importante por su incidencia en el rendimiento. El enfoque metodológico efectuado es básico para definir zonas de manejo dentro de los lotes con el fin de aplicar en forma más eficiente los nutrimentos y de efectuar manejos diferenciales lo cual es un aporte importante a la sostenibilidad y competitividad de la agricultura.

\section{AGRADECIMIENTOS}

Los autores agradecen a la Universidad Nacional de Colombia por el apoyo financiero para la ejecución de esta investigación.

\section{REFERENCIA}

BOOTH, B. Usign ArcGIS 3D analyst. Redlands: ESRI, 2000. 212p.

CRESSIE, N. Statistics for spatial data. New York: John Wiley \& Sons, 1993. 900p.

DIGGLE, P.; RIBEIRO, P. Model based geostatistics. Caxambu: Associação Brasileira de Estatística, 2000. 129p.

FIDALSKI, J.; MARTINS, P.A.; TORMEM, V. Relations among Valencia Orange Yields with Soil and Leaf Nutrients in Northwestern Paraná, Brazil. Brazilian Archives of Biology and Technology, Curitiba, v.43, n.4, p. 387-391, 2000.

FUNAKAWA, S.; YANAI, J.; HAYASHI, Y.; HAYASHI, T.; NOICHANA, C.; PANITKASATE, T.; KATAWATIN, R.; NAWATA, E. Management of tropical sandy soils for sustainable agriculture a holistic approach for sustainable development of problem soils in the tropics. In: SYMPOSIUM ON THE MANAGEMENT OF TROPICAL SANDY SOILS, 1., 2005, Thailand. Proceedings...

GALÁN, S. V. El cultivo de mango. México DF.: Mundi-Prensa, 1999. 299p.

GODWIN, R.; MILLER, C. Review of the technologies for mapping within-field variability. Biosystems Engineering, 
Amsterdam, v.84, n.444 44, p.393-407, 2003.

GOOVAERTS, P. Geostatistics in soil science: state-of-the-art and perspectives. Geoderma, Amsterdam, v.89, p.1-45, 1999.

JIANG, P.; THELEN, K.D. Effect of soil and topographic properties on crop yield in a north-central corn-soybean cropping system. Agronomy Journal, Madison, v.96, p.252-258, 2004.

KRAVCHENKO,A.N.; THELEN, K.D.; BULLOCK, D.G;; MILLAR, N. R. Management, topographical, and weather effects on spatial variability of crop grain yields. Agronomy Journal, Madison, v.97, p.514-523, 2005 .

MARTÍNEZ, L.; ZINCK, A. Modelling spatial variations of soil compaction in the guaviare. ITC Journal, Enschede, n.3, p.252$262,1994$.

MARTÍNEZ, L.; ZINCK, A. Temporal variation of soil compaction and deterioration of soil quality in pasture areas of colombian amazonia. Soil \& Tillage Research, Amsterdam, v.75, n.1, p.3$18,2004$.

MCLEAN, E.O.; HARTWIG, R.C.; ECKERT, D.J.; TRIPLETT, G.B. Basic cation saturation ratios as a basis for fertilizing and liming agronomic crops. II. Field studies. Agronomy Journal, Madison , v.75, p.635-639, 1983.

MIRANDA, D. Evaluación de inductores de la floración en tres cultivares de mango (Mangifera Indica L.). Agronomía Colombiana, Bogotá, v.18, n.2, p.25-30, 2001.

MOTA, M.; RODRÍGUEZ, T.; MONTENEGRO, H.; MARULANDA, S.; CORREA, C.,; BENDECK, M. Métodos analíticos del laboratorio de suelos. 5.ed. Bogotá: IGAC, 1990. 790p.

MUÑOZ, J.; MARTÍNEZ, L.; GIRALDO, R. Variabilidad espacial de propiedades edáficas y su relación con el rendimiento en un cultivo de papa (Solanum tuberosum L.). Agronomía Colombiana, Bogotá, v.24, n.2, p.355-366, 2006.

PEÑA, D. Análisis de datos multivariantes. Madrid: Mc Graw Hill, 2002. 560p.

R DEVELOPMENT CORE TEAM. R: A language and environment for statistical computing. Vienna: R Foundation for Statistical Computing, 2005. Disponível em: 〈http://www.R-project.org>.
REYNIERS, M.; MAERTENS, K.; VRINDTS, E.; BAERDEMAEKER, J. Yield Variability Related to Landscape Properties of a Loamy Soil In Central Belgium. Soil \& Tillage Research, Amsterdam, v.88, p.262-273, 2006.

SILVA, F.M.; MENEZES, Z.; PEREIRA, C.A.; VIEIRA, L.H.; OLIVEIRA, E. Spatial variability of chemical attributes and coffee productivity in two harvests. Ciência Agrotecnica, Lavras, v.32, n.1, p.231-241, 2008.

SUKTHUMRONG, A.; BOONKERD, N.; HUMLERT, R.; FEUNGCHAN, S.; LUKSANAWIMIOL, P.; PRASITTIKNET, J.; SURIYAPAN, O. Plant nutrient and distribution under different fertilizer management in nam dok mai mango. Acta Horticulturae, Belgium, v.509, p.307-314, 2000.

TAYLOR, J.; WOOD, G.; EARL, R.; GODWIN, R. Soil factors and their influence on within-field crop variability: Part II: spatial analysis and determination of management zones. Biosystems Engineering, Amsterdam, v.84, n.4, p. 441-453, 2003.

VRINDTS, E.; REYNIERS, M.; DARIUS, P.; BAERDEMAEKER, J.; GILOT, M.; SADAOUI, Y.; FRANKINET, M.; HANQUET, B.; DESTAIN, M.F. Analysis of Soil and Crop Properties for precision agriculture for winter wheat biosystems. Engineering, Amsterdam, v.85, n.2, p.141-152, 2003.

WEBSTER, R. Quantitative spatial analysis of soil in the field. Advances in Soil Science, New York, v.3, p.1-70, 1985.

WILDING, L.; DREES, L. Spatial variability and pedology. In: WILDING, L.; SMECK, N.E.; HALL, G.F. (Ed.). Pedogenesis and soil taxonomy: concepts and interactions. Amsterdam: Oxford University Press, 1983. v.1, p.83-113

YAHIA, E.; ORNELAS, J.; ARIZA, R. EI mango. México: Trillas, 2006, 244p. 